\title{
EFFECT OF SYSTEMIC ADMINISTRATION OF SILVER NANOPARTICLES IN TREATMENT OF EXPERIMENTAL PERIODONTITIS IN ALBINO RATS
}

\author{
Waleed Mohamed Shaker ${ }^{1}$, Enas Mahmoud Hegazy ${ }^{2}$, Laila Sadek Ghali ${ }^{3}$
}

DOI: $10.21608 / d s u .2021 .60867 .1065$

Manuscript ID: DSU-2102-1065

\section{KEYWORDS}

Albino rats - Histological

- Periodontitis - Silver

nanoparticles Ultrastructural.

- E-mail address:

Dr_waleed@dent.svu.edu.eg

1. Assistant Lecturer in Oral Biology Department, Faculty of Dentistry, South Valley University

2. Associate Professor of Oral Biology, Faculty of Dentistry, Suez Canal University

3. Professor of Oral Biology, Faculty of Oral and Dental Medicine, Cairo University

\begin{abstract}
Introduction: Periodontal disease is one of the main dental diseases that affect millions of people around the world. Modern nanotechnology progression and renovations through nanodentistry providing a suitable solution for the treatment of many dental illnesses including periodontal disease. Aim: Histological and ultrastructural evaluation of the effect of silver nanoparticles in treatment of periodontitis in albino rats. Materials and methods: A total of 18 adults male albino rats with body weight ranging from 150 to $180 \mathrm{gm}$ were used and divided into three groups group 1: served as control, group 2: used for induction of experimental periodontitis using wiring around the cervix of mandibular central incisors for two weeks, and group 3: after induction of experimental periodontitis as in group 2 they were subjected to intraperitoneal injection with silver nanoparticles for the treatment of periodontitis three times per week for four weeks. Results: Histological and ultrastructural investigation revealed that periodontal ligament around ligated mandibular incisors showed marked improvement in the degenerated fibers after injection with silver nanoparticles in group 3 compared to group 2 as the fibers regained their association in strong bundles and they regained their attachment to regenerated bone and tooth surface. Conclusions: Marked regeneration of the periodontium of periodontally affected ligated mandibular incisors reflected that silver nanoparticles have a great anti-inflammatory effect
\end{abstract}

\section{INTRODUCTION}

Periodontal diseases have been and still are vague both to patients and to doctors. Periodontal disease is widespread disease for which microorganism infections have been identified as the main etiology. Periodontitis is an inflammatory disease which affects adult humans. The disease destroy the periodontium i.e., tooth-supporting tissues as periodontal ligament, gingiva and alveolar bone ${ }^{(\mathbf{1})}$.

No single line of treatment is enough to overcome the deterioration effects of periodontal diseases. Taking Nano-dentistry as a promising innovation to improve dental conditions and oral hygiene has made researchers to find new methods which expand their knowledge and techniques to make new drug delivery systems and dental Nano-materials. The unique physico-chemical properties of silver nanoparticles when combined with the inhibitory capacity against the 
growth of microorganisms lead to an upsurge in the research on NPs and their potential application as antimicrobials ${ }^{(2)}$.

Silver nanoparticles, with diameter ranging from tens to hundreds of nanometers, have unique properties that differ from bulk material features. Changes in its physical, chemical, mechanical, thermal as well as electrical, magnetic, and luminescent properties is attributed to the increased relative surface area of nanoparticles (NPs), compared with fine and ultrafine particle bulk material $^{(3)}$.

It was suggested that the antimicrobial mode of action of Ag-NPs is due to the formation of free radicals and free radical-induced membrane damage. The free radicals may be derived from the surface of Ag-NPs and be responsible for the antibacterial activity. Moreover, it was reported that the attachment of Ag-NPs to the cell surface disturb permeability and respiratory functions of the cell ${ }^{(4)}$.

In this investigation we are looking for a novel line of treatment of periodontitis using silver nano particles due to their promising antibacterial and anti-inflammatory properties.

\section{MATERIALS AND METHODS}

The study was conducted after the approval of Ethical Committee, Faculty of Dentistry, Suez Canal University (No 40/2017)

\section{Drug preparation:}

\section{Preparation and characterization of the Nano particles:}

Rapid green method was used for the preparation of silver nanoparticles as follow ${ }^{(5)}$.

\section{Pomegranate extract preparation}

- Pomegranate fruits were bought from the market. All glass wares and the pomegranate fruits were washed thoroughly with distilled water. The glass wares were dried in hot air oven.

- The peel was removed from the washed pomegranate fruit and washed again with deionized water and air dried. $100 \mathrm{ml}$ of distilled water were added to 20 gm of pomegranate fruit peel was weighted and added in in 250 $\mathrm{ml}$ Erlenmeyer flask and boiled for 10 minutes. Filtration of the boiled materials was done to get aqueous fruit peel extract which was used for silver nanoparticles synthesis.

- Computerized analytikjenaSPECORD 200 Plus double beam spectrophotometer (Germany) was used for studying the optical properties at room temperature in the wavelength range 190 - $1100 \mathrm{~nm}$.

\section{Silver nanoparticles preparation}

$1 \mathrm{mM}$ aqueous solution of silver nitrate was prepared for $100 \mathrm{ml}$. To this $5 \mathrm{ml}$ of pomegranate extract was added and incubated for 24 hours with intermittent shaking. After 24 hours the color change to brown indicated the formation of silver nanoparticles.

The bio reduction of $\mathrm{Ag}+$ ion in aqueous solution was watched with the help of UV-visible spectroscopic analysis. Figure 1, shows the ultraviolet visible spectrum of silver nanoparticles. A characteristics surface plasmon absorption bands were observed at $271 \mathrm{~nm}$ after $24 \mathrm{hrs}$ incubation, which confirm that the prepared material is in nano form with particle size about $40 \mathrm{~nm}$. 


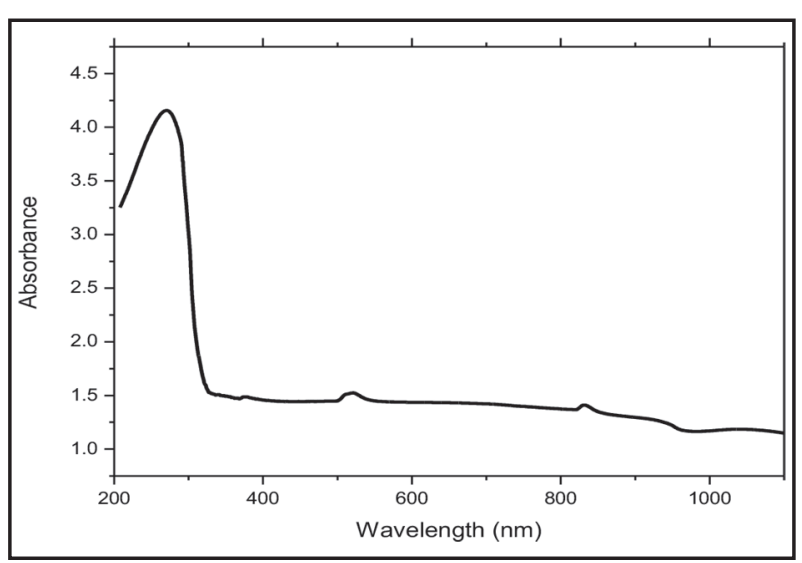

Fig. (1) UV - Vis absorption spectra for the prepared AgNPs.

\section{Study design:}

A total of 18 adult male albino rats with body weight ranging from 150 -180 grams were used in the study; The rats were housed in wire cages, in a temperature and humidity controlled room $(23 \pm$ $1{ }^{\circ} \mathrm{C}$ and $60 \pm 5 \%$ relative humidity), under 12 -hour light/dark cycle. all animals were fed natural diet throughout the whole experimental period which lasted for six weeks in the animal house, Faculty of Dentistry, Suez Canal University.

Animal grouping: The animals were divided as follows:

Group 1 (control group): Consisted of $\mathbf{6}$ animals, were injected intraperitoneally with distilled water and served as controls.

Group 2 (periodontitis group): Consisted of 6 animals. Ligatures with figure "8", 4/0 nonresorbable sterile silk thread were placed around the cervix of right and left mandibular central incisors under general anesthesia. General anesthesia was achieved through IM injection with Ketamine $10 \%$ and Xylazine 2\% (2:1), $0.12 \mathrm{ml} / 100 \mathrm{~g}$ body weight. The animals were left for 2 weeks for induction of periodontitis ${ }^{(6)}$. They were subjected to intraperitoneal injection with distilled water three times a week during the time of the experiment which lasted for 4 weeks (the total time of the experiment was 6 weeks).

Group 3 : Nano silver treated (NS group): Consisted of $\mathbf{6}$ animals, induction of periodontitis was carried out as in group 2. After two weeks the animals were subjected to intraperitoneal injection with silver Nano-particles (AgNPs) (the particles size of about $40 \mathrm{~nm})$ at a dose of $10 \mathrm{mg} / \mathrm{kg}(0.5$ $\mathrm{mg} / \mathrm{ml}$ in distilled water) three times a week for 4 weeks $^{(7)}$.

Finally, the animals of different groups were anesthetized through IM injection with a solution of Ketamine 10\% and Xylazine 2\% (2:1), $0.12 \mathrm{ml}$ $/ 100 \mathrm{~g}$ body weight and euthanized by cervical dislocation. Their jaws were dissected out.

\section{Tissue processing for histological and ultrastruc- tural examination:}

- Half of the specimens were fixed in $10 \%$ neutral buffered formalin, decalcified in $10 \%$ Ethylenediaminetetraacetic acid solution. After complete decalcification, the specimens will be processed, and embedded in paraffin. Six microns thick sections were cut and subjected to Hematoxylin and Eosin stain for histological examination and detection of any structural changes.

- The other half were immediately fixed in 3\% glutaraldehyde, after complete decalcification in $10 \%$ EDTA solution, very small sample $\left(1 \mathrm{~mm}^{3}\right)$ were taken from the investing tissues of the incisors region in the mid root area, fixed in osmium tetroxide, processed, and embedded in epoxy resin . Ultrathin sections were cut and stained with urenyl acetate and lead citrate for transmission electron microscope examination in the faculty of Agriculture Cairo University. 


\section{RESULTS}

\section{I- Histological results: [Hematoxylin and eosin result]:}

\section{Group 1 (control group):}

- The periodontal ligament of the control rats was composed of cells, fibers and blood vessels. The collagen fibers which are the main components of the PDL were arranged in a parallel manner in bundles attaching the tooth to the alveolar bone. Fig.2-A

- Cells of the PDL were predominantly fibroblasts and progenitor cells. Some of the defensive cells were seen.

The alveolar bone showed normal turnover rate, as the number of the reversal lines were minimal. Besides, the periodontal ligament (PDL) bone interface appeared to be smooth; no osteoclastic activity was observed. Fig.2-B

Gingiva showed normal keratintized epithelium and lamina propria with blood vessels and few inflammatory cells could be seen. Fig.2-C

\section{Group 2 (periodontitis group):}

Periodontal ligament of rats mandibular incisors with wire ligatures left around them for two weeks for induction of periodontitis showed degenerative changes in the periodontal ligament in the form of disorientation, dissociation, and detachment of the principle fibers Fig.3-A, infiltration of inflammatory cells, marked dilatation of blood vessels, in addition to lots of Howship's lacunae and osteoclastic activity on the surface of the bone and widening of the marrow cavities.Fig.3-B

Gingiva showed hyperkeratinization, sometimes atrophy and/or ulceration of surface epithelium with cytoplasmic swelling and vacuolization and marked dilatation of blood vessels of the lamina propria. Fig. 3-C.

\section{Group 3 (AgNPs):}

Periodontal ligaments of rats incisors of Group 3 that were subjected to induction of periodontitis in the same way as in group 2 animals, for 2 weeks and were subjected to intraperitoneal injection with silver Nano-particles (AgNPs) (the particles size of about $40 \mathrm{~nm}$ ) at a dose of $10 \mathrm{mg} / \mathrm{kg}$ (each $0.5 \mathrm{mg}$ was dissolved in one $\mathrm{ml}$ of distilled water) three times a week for one month, showed improvements, mostly in the arrangement and association of the fibers of PDL. Compared to periodontitis group, the fibers mostly regained their arrangement and association together in bundles, as dissociation of fibers and disorientation were nearly absent in this group of animals. Dilatation of the blood vessels was still

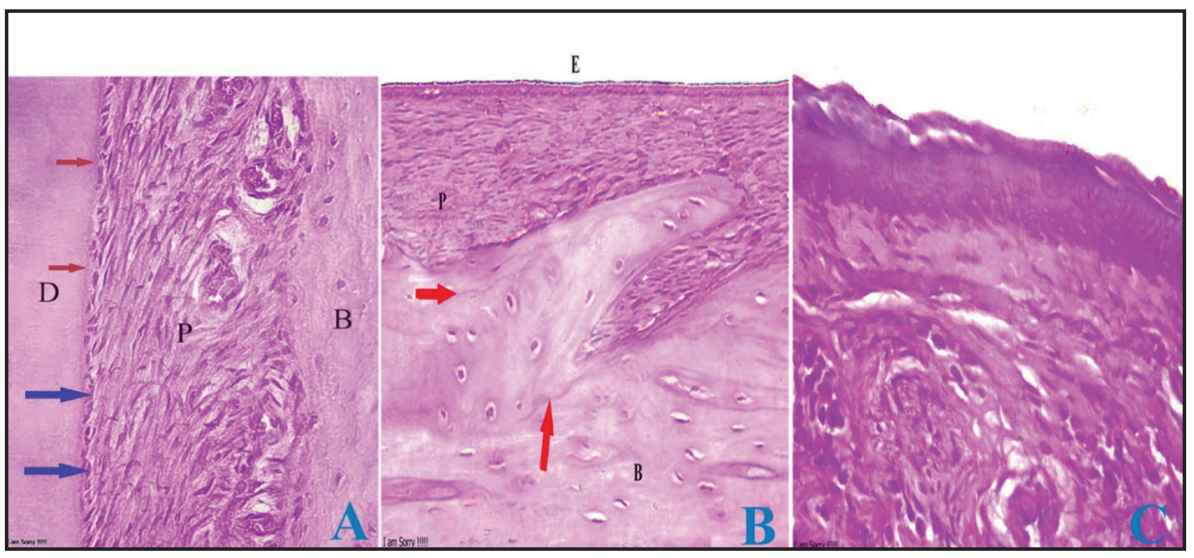

Fig. (2) Showing periodontal ligament fibers $(\mathrm{P})$ between dentin (D) and alveolar bone (B) (fig.2-A), periodontal ligament fibers on labial surface (fig. 2-B) and gingival keratinized stratified squamous epithelium (fig.2-C) 
observed, however at a minimal degree compared to group 2 animals., while on the root side cementum showed new cementoid tissue formation. Fig.4A\&B

Also gingival tissue showed marked regeneration as surface epithelium appeared normal with no ulceration or vacuolization and lamina propria appeared normal with minimal dilatation of blood vessels and infiltration of inflammatory cells fig.4-C.

\section{II-Electron Microscopic Results:}

Group 1 (control group): The electron microscopic (EM) examination of the PDL of this group revealed the normal ultrastructure of the collagen bundles that were either cut longitudinally or transversely extending between the bone and tooth structure. According to the direction of section, the fibroblasts were either spindle, stellate or rounded in shape the fibroblasts were presented with large rounded or oval nuclei, normal rough endoplasmic reticulum (RER), Golgi apparatus and mitochondria. Fig.5-A.

No attachment epithelium was presented since the sample was taken from the mid root area Alveolar bone showed normal ultrastructure of bone cells osteoblasts and osteocytes in their lacunae.Fig.5-B.
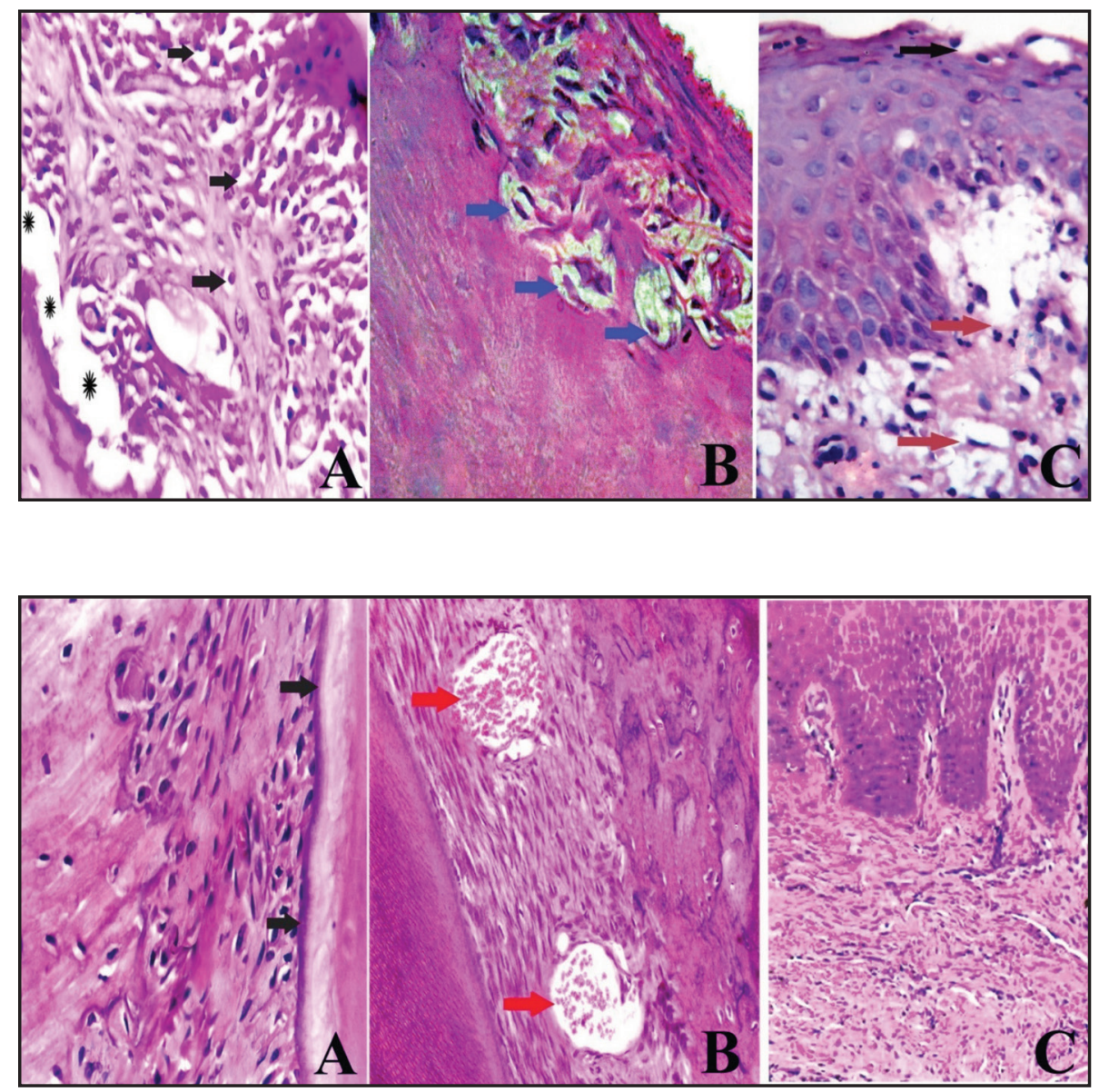

Fig. (3) Showing degenerative changes in the form of disorientation, dissociation, and detachment of the principle fibers with a lot of inflammatory cells infiltration (A), osteoclastic activity with osteoclasts in their Howship's lacunae (B) and atrophy of surface epithelium with cytoplasmic swelling and vacuolization and marked dilatation of blood vessels of the lamina propria (C).
Fig. (4) The fibers mostly regained their arrangement and association together in bundles, the root side cementum showed new cementoid tissue formation (black arrows) (A). Dilatation of the blood vessels at a minimal degree (B), gingival tissue showed normal epithelium with no ulceration or vacuolization and lamina propria appeared normal with minimal dilatation of blood vessels and infiltration of inflammatory cells $(\mathrm{C})$. 


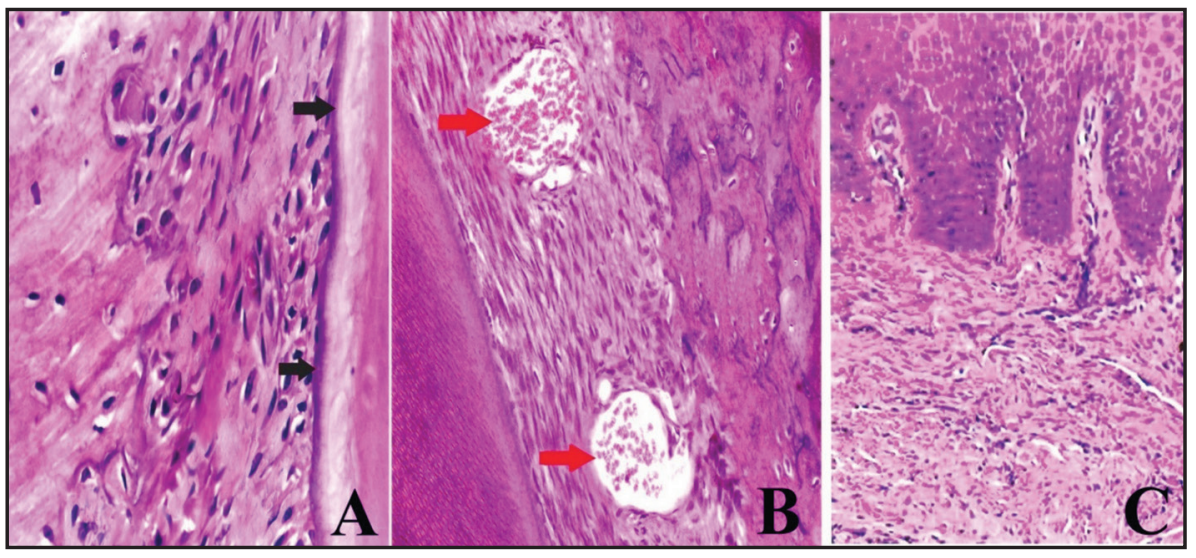

Fig. (5) Showing normal fibroblast (F) with nrmal arrangement of fibers (C) (Fig.5-A) and normal osteocyte in lacuna (B).

\section{Group2 (periodontitis Group):}

EM examination of the PDL and alveolar bone of this group ensured the histopathological findings where there were marked destruction and marked reduction in density of the collagen fibers of PDL leaving a lot of debris. The fibroblast cells showed definite signs of degeneration as they were markedly shrunken with pyknotic nuclei, degenerated organelles, vacuolated cytoplasm and fatty infiltration. Also dilatation of blood vessels was observed. Fig. 6-A

An apparent increase in the number of lymphocytes, plasma cells and eosinophils with signs of degenerations was so marked.Fig.6-B.
Osteoclastic activity of the alveolar bone was clear with osteoclasts on bone surface and osteocytes appeared atrophic with pyknotic nuclei Fig.6-C.

\section{Group 3. (treated with nanosilver) :}

The electron microscopic examination of the PDL of this group showed moderate regenerative changes in the fibrous and cellular elements. Fibroblasts appeared almost normal with normal nucleus, cytoplasm and cell organells showed normal mitochondria, rough endoblasmic reticulum and Golgi apparatus Fig.7-A. Collagen fibers regaind their orientation and arrangement in strong bundles.Fig.7-B

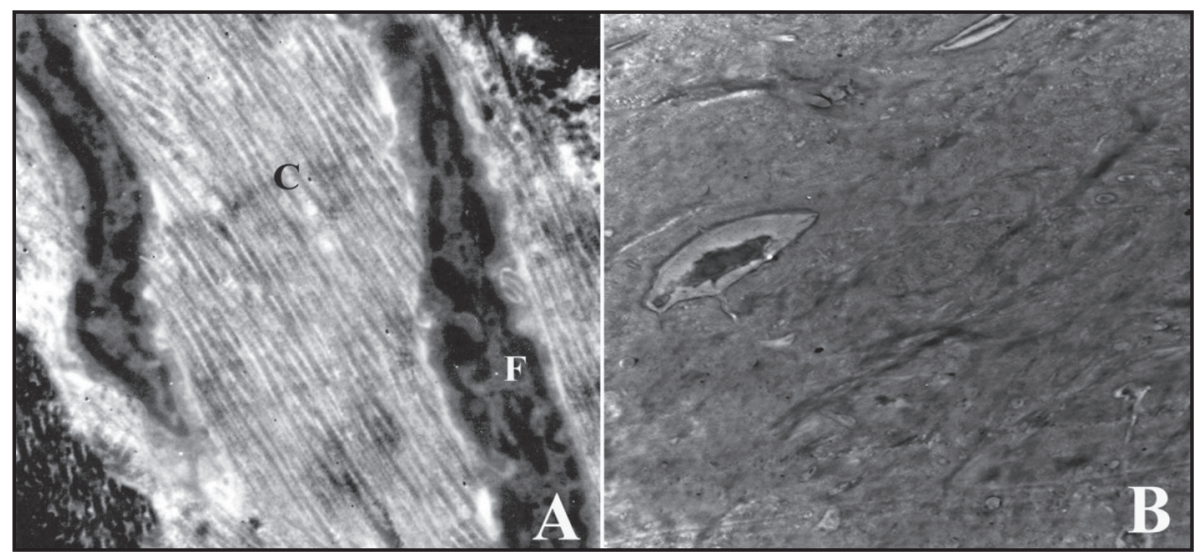

Fig. (5) Showing normal fibroblast (F) with nrmal arrangement of fibers (C) (Fig.5-A) and normal osteocyte in lacuna (B). 


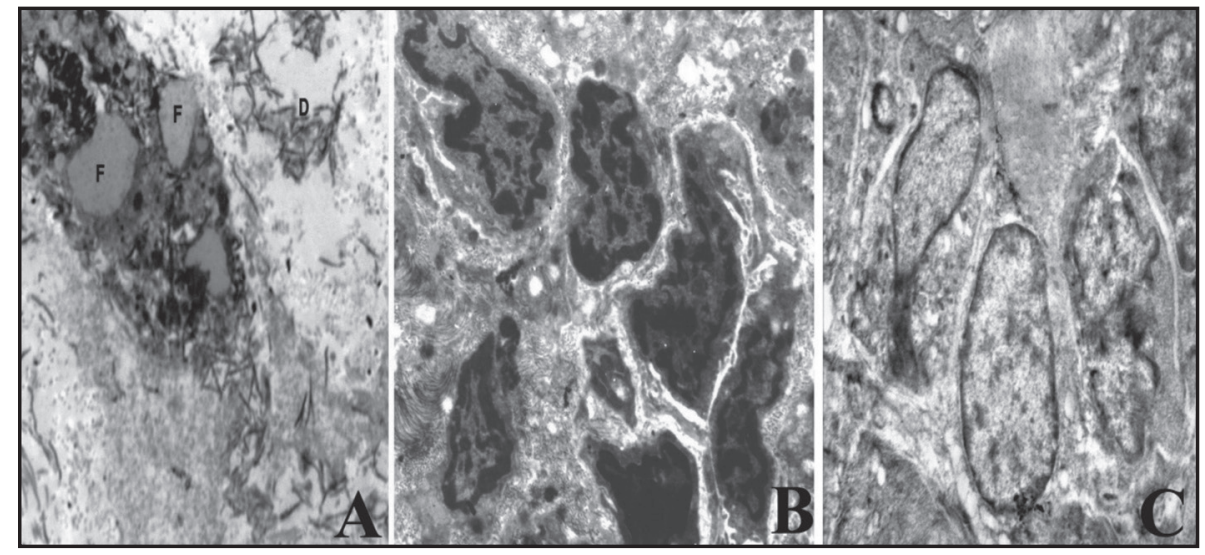

Fig. (6) Showing degenerative changes in fibroblasts (f) and collagen fibers (D) (Fig.6-A) with inflammatory cells infiltration (B) and osteoclast (C).

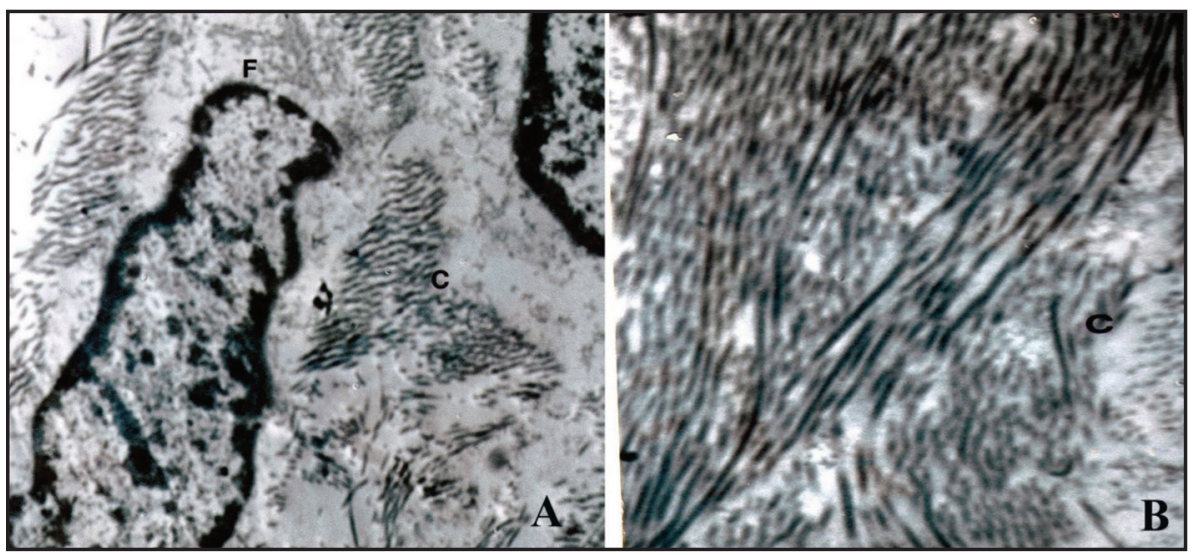

Fig. (7) Showing regeneration of periodontal cells (A) and fibers (B).

\section{DISCUSSION}

Periodontitis is a chronic inflammation of the tooth supporting structures that results in periodontal tissue destruction and alveolar bone loss. Tissue destruction occurs as a result of the host's attempt to eliminate bacteria from the gingival sulcus by stimulating an immuno-inflammatory response ${ }^{(8)}$. The aim of periodontal therapy is to decrease the pathogenic bacterial nomber to the level at which the periodontal destruction is stopped ${ }^{(9)}$. It was reported that the nonsurgical treatment can reduce inflammation, decrease the pocket depth and regain of attachment. However, mechanical therapy alone may fail to overcome the pathogenic bacteria because of their deep location within gingival tissues or in other areas inaccessible to periodontal instrumentation ${ }^{(\mathbf{1 0})}$.
Although an additional clinical benefit was reported when systemic antibiotics have been described, it is only preferred in cases of aggressive or refractory types of periodontitis to prevent the development of antibiotic resistant bacteria ${ }^{(11)}$.

Nanotechnology has extended into all fields of science; it displays important alternative ways to solve scientific and medical questions and problems one of them is the antibacterial resistance against antibiotics ${ }^{(12)}$.

Nanoparticles (NPs) displayed superior physical, chemical, mechanical, thermal and biological features as compared to the bulk materials. Some of these important to the biological and research fields are their anti-microbial, anti-inflammatory, antioxidant and anti-angiogenic properties ${ }^{(13)}$. 
Nanoparticles were used in these fields due to their superior properties. The new modality is the development of nanoparticles which have better therapeutic properties and not harmful to the environment ${ }^{(14)}$. Oeganic synthesis of nanoparticles eliminate the need of employing toxic materials, decrease cost and protect the environment by excluding toxic materials as reducing and capping agents $^{(15)}$.

Organic synthesis of Nanoparticles include the use of various organic sources as plant, bacteria, fungus, yeast, and viruses ${ }^{(16)}$. Green synthesis of nanoparticles using plants is considered as one of the most preferred options for nanoparticle synthesis because it requires a neutral $\mathrm{pH}$ and takes place at room temperatures ${ }^{\left({ }^{17}\right)}$. That is why we used the green method in synthesizing silver nanoparticles and it was not used only by us but also used by Shanmugavadivu M.,et al who used pomegranate extract as a green method for the synthesis of metal nanoparticles ${ }^{(7)}$.

Antimicrobial nanoparticless have wide ranges of applications in dentistry, they have a significant antibacterial effect, but their antibacterial properties depends on the concentration, size, form and other factors ${ }^{(14)}$.

In the present investigation Induction of experimental periodontitis using ligature wires around rat's mandibular incisors for two weeks in group 2 was not done only by us, but also by Ionel A. et al ${ }^{(6)}$.

The present histopathological results reflected degenerative changes in the periodontal ligament, infiltration of inflammatory cells, marked dilatation of blood vessels, in addition to osteoclastic activity on the surface of the bone and widening of bone marrow cavities. Gingiva showed hyperkeratinization, sometimes atrophy and/or ulceration of surface epithelium with cytoplasmic swelling and vacuolization and marked dilatation of blood vessels of the lamina propria.

These results were ensured by electron microscopic examination that showed marked destruction and marked reduction in density of the collagen fibers of PDL leaving a lot of debris. The fibroblast cells showed definite signs of degeneration as they were markedly shrunken with pyknotic nuclei, degenerated organelles, vacuolated cytoplasm and fatty infiltration.

An apparent increase in the number of lymphocytes, plasma cells and eosinophils with signs of degenerations was so marked. Osteoclastic activity of the alveolar bone was clear with osteoclasts on bone surface and osteocytes appeared atrophic with pyknotic nuclei.

Our results coincided with those of Ionel A. et al. who stated that modified "ligature" model of periodontitis in rats has many advantages: short time of induction of periodontal disease (only 2 weeks), clear clinical signs of inflammation of periodontal tissues and resorption of the alveolar bone, and simple method for induction of periodontal disease ${ }^{(}{ }^{(}$.

In the present investigation the rats received 10 $\mathrm{mg} / \mathrm{kg}$ body weight (each $0.5 \mathrm{mg}$ was dissolved in one $\mathrm{ml}$ of distlled water) 3 times per week for 4 weeks of silver nanoparticles with particle size of about $40 \mathrm{~nm}$ intraperitoneally to study their effect on periodontitis in rats that was induced using ligature wires around their mabdibular incisors for 2 weeks as in group 2.

Silver was known as an antimicrobial component in therapeutic wound treatment for along time. It can promote wound healing and have strong antiinflammatory properties ${ }^{\left({ }^{(18)} \text {. }\right.}$ 
In group 3 animals that were injected intraperitoneally with AgNPs our histological results showed regenerative changes and improvements, mostly in the arrangement and association of the fibers of PDL. Alveolar bone showed regenerative changes in the form of new osteoid tissue formation and absence of osteoclastic activity.

Also gingival tissue showed marked regeneration as surface epithelium appeared normal with no ulceration or vacuolization and lamina propria appeared normal with minimal dilatation of blood vessels and infiltration of inflammatory cells.

Our ultrastructural results showed regenerative changes in the fibrous and cellular elements. Fibroblasts appeared almost normal with normal nucleus, cytoplasm and cell organells showed normal mitochondria, rough endoblasmic reticulum and Golgi apparatus. Collagen fibers regaind their orientation and arrangement in strong bundles. Alveolar bone showed normal ultrastructure with osteocytes in their lacunae.

Our findings coincided with those previously reported by Heba A. Shawky et al. who found marked improvement in clinical manifestations and decrease in microbial infection after intrasulcular injection of silver nanoparticles asits effect was similar to tetracycline films application in treatment of periodontal pockets ${ }^{(11)}$.

The reduction of inflammation in the periodontal ligament and gingiva of Group 3 animals could be due to the antibacterial activity of nanosilver which plays a significantt role in reducing inflammation.

This suggestion was the same reported by Nadworny et al. who stated that silver nanoparticles had direct anti-inflammatory effects.

Ag-NPs have an antibacterial activity against both Gram positive and gram negative bacteria. This effect depends on its ions, that bind to sulfur, oxygen or nitrogen in biological molecules having electron donor groups. It has also been reported that silver ions can inhibit respiratory enzymes lwading to the production of reactive oxygen species (ROS), that may attack the bacterial cell itself ${ }^{(\mathbf{1 9})}$.

Another mode of action of silver ions is prevention of bacterial cell multiplication by interaction with their DNA. Nanoparticles can act on soft bases in DNA as sulfur and phosphorus as its main components and destroy it. This can inhibit DNA replication of the bacteria leading to cell death and can modify bacterial transduction signals ${ }^{(11)}$.

It is a well-known that phosphorylation of bacterial protein substrates affects transduction signal in bacteria. Dephosphorylation of tyrosine residues is observed in Gram-negative bacteria. Alteration of phosphotyrosine profile of bacterial peptides by nanoparticles was observed. It has been reported that the nanoparticles dephosphorylation of the peptide substrates on tyrosine residues by NPs, that leads to growth inhibition by inhibiting transduction of bacterial signal ${ }^{(20)}$.

\section{CONCLUSIONS}

Depending on the results of this study silver nanoparticles have a promising anti-inflammatory effect that can help in overcome the deleterious effects of periodontitis.

\section{REFERENCES}

1. Sowjanya G, Mallanagouda BP, Mehta DS. Rat as laboratory animal model in periodontology. Int J Oral Health Sci. 2017; 7: 69-75.

2. Iadiz MAR, Bamedi M, Fakour SR. Periodontal diseases and recently applied nano- technology: A review article. Health Sci J. 2017; 9: 345-351.

3. Moghimi SM, Kissel T. Particulate nanomedicines. J Adv Drug Deliv Rev. 2006; 58:1451-1455. 
4. Sondi IB, Salopek-Sondi J. Silver nanoparticles as antimicrobial agent: a case study on E. coli as a model for gram-negative bacteria. J Colloid Interface Sci. 2004; 275: 177-182.

5. Shanmugavadivu M, Selvam K, Ranjithkumar R. Synthesis of pomegranate peel extract mediated silver nanoparticles and its antibacterial activity. Am J Alzheim. 2014; 2: 174-182.

6. Ionel A, Lucaciu O, Moga M, Buhatel D, Ilea A, Tabaran $\mathrm{F}$, et al. Periodontal disease induced in Wistar rats - experimental study. J HVM. 2015; 7: 90-95.

7. BorisA K, Larisa IP, Vladimir BG, Oleg HM. Comparative in vivo assessment of some adverse bioeffects of equidimensional gold and silver nanoparticles and the attenuation of nanosilver's effects with a complex innocuous bioprotectors. Int J Mol Sci. 2013; 14: 2449-2483.

8. Ji S, Choi Y. Innate immune response to oral bacteria and the immune evasive characteristics of periodontal pathogens. JPIS. 2013; 43:3-11.

9. Knöfler GU, Purschwitz RE, Eick S, Pfister W, Roedel M, Jentsch HF. Microbiologic findings 1 year after partial- and full-mouth scaling in the treatment of moderate chronic periodontitis. Quintessence Int J. 2011;42:107-17.

10. Banodkar AB, Rao J. A comparative study of periodontal treatment using tetracycline impregnated collagen fibers as compared to scaling and root planing alone-A clinical and microbiological study. JIDA. 2011; 5: 1044-46.

11. Shawky HA, Basha SM, EL Batouti GA, Kassem AA. Evaluation of clinical and antimicrobial efficacy of silver nanoparticles and tetracycline films in the treatment of periodontal pockets. J appl dent. 2015; 4: 113-123.
12. Agarwal H, Nakara A, Shanmugam VK. Anti-inflammatory mechanism of various metal and metal oxide nanoparticles synthesized using plant extracts: A review. Biomed Pharmacother. 2019; 109 : 561-572.

13. Phull AR, Abbas Q, Ali A, Raza H, kim S, Zia JM. Antioxidant, cytotoxic and antimicrobial activities of green synthesized silver nanoparticles from crude extract of Bergenia ciliata, FJPS. 2016; 2: 31-36.

14. De Crozals G, Bonnet R, Farre C, Chaix C. Nanoparticles with multiple properties for biomedical applications: a strategic guide. J Nan Today. 2016; 11: 435-463.

15. Luo J. Mechanical properties of nanoparticles: basics and applications. J Appl Phys. 2014; 47: 3001- 3003.

16. Shah M, Fawcett D, Sharma S, Tripathy SK, Poinern GEJ. Green Synthesis of Metallic Nanoparticles via Biological Entities. J Nanotechnol. 2015; 11: 2-9.

17. Velusamy P, Kumar GV, Jeyanthi V, Das J, Pachaiappan R. Bio-inspired green nanoparticles: synthesis, mechanism, and antibacterial application. J Toxicol Res. 2016; 32: 95-102.

18. Kenneth KYW, Stephanie OFC, Huang L, Jun N, Chang $\mathrm{T}$, Chi-Ming $\mathrm{H}$, et al. Further Evidence of the Antiinflammatory Effects of Silver Nanoparticles. Chem Med Chem. 2009; 4: 1129 -1135.

19. Morones JR, Elechiguerra JL, Camacho A, Holt K, Kouri JB, Ramirez JT, et al. The bactericidal effect of silver nanoparticles. J Nanotechnol. 2005; 16 : 2346-2353.

20. Shrivastava S, Bera T, Roy A, Singh G, Ramachandrarao P, Dash D. Characterisation of enhanced antibacterial effects of novel silver nanoparticles. J Nanotechnol. 2007; 18: 1-9. 